\title{
Experimental investigation of the inflection geometry on dual bell nozzle flow behavior
}

\author{
Chloé Génin* and Ralf Stark ${ }^{\dagger}$ \\ German Aerospace Center, Lampoldshausen, D-74239, Germany
}

\begin{abstract}
The altitude adaption of the dual bell nozzle is achieved by the contour inflection, leading to a symmetrical and controlled separation under sea level conditions. When reaching a certain pressure ratio, the transition to high altitude mode takes place and the nozzle flows full. Former experimental studies have shown the existence of an intermediate flow state before the transition from one operating mode to the other takes place: the sneak transition, characterized by the separation of the flow downstream to the inflection, leading to increased side loads. To minimize this effect, various contour inflection geometries have been investigated. The study was divided into a cold flow and a warm flow test campaign on sub-scale planar models.
\end{abstract}

\section{Nomenclature}

$\begin{array}{ll}\alpha & \text { Inflection angle } \\ \epsilon & \text { Area ratio } \\ L & \text { Length, mm } \\ N P R & \text { Nozzle Pressure Ratio, } P_{0} / P_{a} \\ P & \text { Pressure, MPa } \\ T & \text { Temperature, } \mathrm{K}\end{array}$

Subscript

$\begin{array}{ll}0 & \text { Total conditions } \\ a & \text { Ambient } \\ b & \text { Base nozzle } \\ e & \text { Extension nozzle } \\ i & \text { Inflection region } \\ w & \text { Wall }\end{array}$

\section{Introduction}

The European heavy lifter Ariane 5 features a parallel staged design, where a cryogenic main stage is supported by two solid boosters generating the main part of the lift-off thrust. The objective of Ariane 5 is the dual GTO payload capability. Its main stage engine Vulcain 2 has to be ignited on the ground for security reasons to assure proper running before solid boosters' ignition and rocket take off. Because of this constraint, the main stage engine has to fulfill a wide range of operation conditions, from sea level to near vacuum. Flow separation in the nozzle has to be avoid at sea level to reduce undesired side loads that would affect the engine, the rocket structure and even the payload itself. The resulting area ration limitation leads to performance losses: at low altitude, the engine exhaust flow is driven over expanded and at high altitude, highly under expanded.

Altitude adaptive nozzle concepts generate a thrust that is not only optimized for one specific altitude. The overall specific impulse $I_{s p}$ of the engine can be hence increased over the ascent. Therefore, they have come into focus as the sub system with the most promising performance gain. Different concepts

\footnotetext{
*Research scientist, Nozzle group, Institute of Space Propulsion.

${ }^{\dagger}$ Head of Nozzle group, Institute of Space Propulsion.
} 
were developed to circumvent the limitation in area ratio of conventional nozzles. The commonly discussed solutions are plug, extendible and dual bell nozzles.

The characteristic contour inflection of the dual bell nozzle divides the nozzle into base and extension (Fig. 1) and offers a one-step altitude adaptation. The concept exists in the literature since 1949, proposed in a study by Foster and Cowles, ${ }^{1}$ but the first investigations were conducted only in the early 1990's (for example by Horn and Fisher ${ }^{2}$ or Hagemann et al. ${ }^{3}$ ).
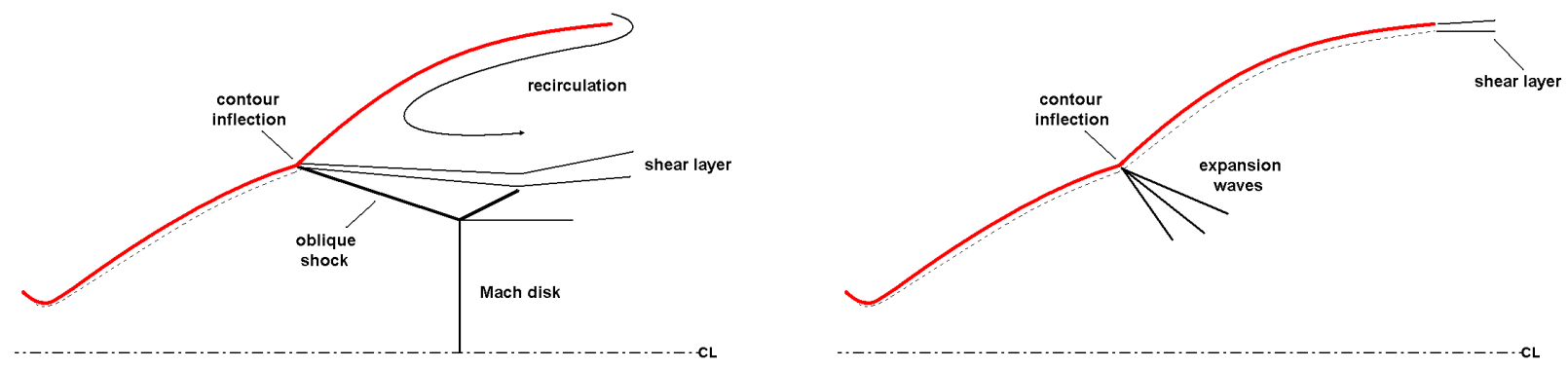

Figure 1. The two operation modes of a dual bell nozzle: sea level mode (left) and altitude mode (right).

Under sea level conditions, the flow separates at the contour inflection in a controlled and symmetrical way. The side load generation is kept reduced and the thrust increased due to the small area ratio. During flight, the ambient pressure decrease, increasing the nozzle pressure ratio $\left(\mathrm{NPR}=P_{0} / P_{a}\right)$. At a certain altitude, the transition NPR is reached and the separation point leaves the contour inflection and moves rapidly (in a few milliseconds) to the nozzle exit. The extension flows then full. The altitude thrust is increased due to the larger area ratio of the extension.

The transition from sea level to high altitude mode is preceded by the sneak transition. The phenomenon has been pointed out in earlier studies ${ }^{4}$ conducted at the German Aerospace Center (DLR). It corresponds to an intermediate state, taking place when increasing the NPR, before the start of the actual transition. The separation point leaves the contour inflection and starts moving down the extension, in the region close to the inflection, where the wall pressure gradient is negative. The sneak transition is comparable to the separated flow in a conventional nozzle and leads to increased side loads ${ }^{5}$.

The sneak transition has to be limited in length, i.e. the region of negative wall pressure gradient in the extension, and in duration, i.e. the NPR interval between the instant, when the separation leaves the contour inflection and the start of the transition. A potential solution is to optimize the geometry of the contour inflection. Therefore, five different geometries have been designed and tested at the DLR Lampoldshausen.

A planar nozzle model with exchangeable contour has been chosen. The contoured sides are exchangeable and offer five geometrical concepts. All of the contours were tested under cold flow conditions and the three most promising were then tested under warm flow conditions.

\section{Experimental setup and procedure}

\section{II.A. Test facilities}

The study was divided into two test campaigns, the first one conducted at the cold flow facility P6.2 and the second at the warm flow test complex M11 at DLR Lampoldshausen.

The P6.2 facility was designed for the study of subscale nozzles, diffusers and ejector setups. The horizontal test position offer sea level test environment.

Feeding gas is dry nitrogen. It is stored in high pressure vessels under ambient conditions. The maximum feeding pressure of the facility is $6 \mathrm{MPa}$. Up- and down-ramping of the feeding pressure $P_{0}$ leads to the flow transition from one operating mode to the other. Figure 2 is a picture of the nozzle model mounted on the test facility.

The test position M11.1 was designed for the study of nozzle models and scramjets. The feeding gas is a mixture of air and water damp (about $95 \%$ to $5 \%$, depending on the operation point). Under pressure air flows through oxygen-hydrogen burners and can be heated up to a total temperature of $T_{0}=1300 \mathrm{~K}$. The maximum feeding pressure reached at M11.1 facility is $P_{0}=3 M P a$. Pressure and temperature are adjusted by varying the air, oxygen and hydrogen mass flows. The test conditions are steady state ${ }^{6}$ and the 


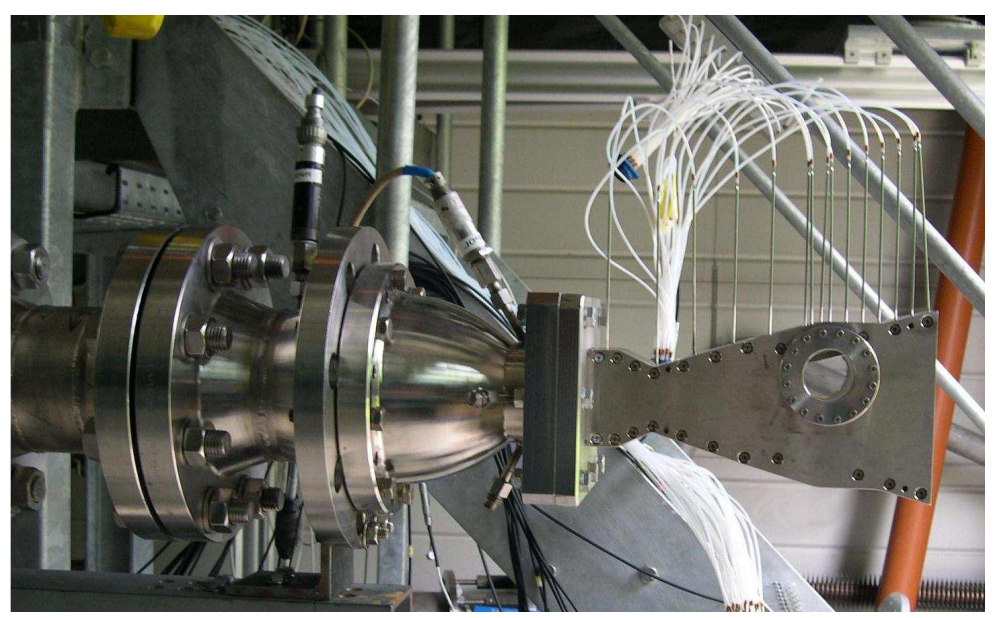

Figure 2. Planar dual bell nozzle mounted on P6.2 test facility.

test duration can be set between $5 \mathrm{~s}$ and $60 \mathrm{~s}$.

\section{II.B. Nozzle models}

For this study of the inflection region, a planar nozzle model has been chosen to permit the observation of the flow in the vicinity of the inflection. The model was constituted of two side plates and two contoured sides. The lower contour corresponds to the classical sharp edge inflection. The upper contour is exchangeable and offers different geometries. The first contour yields as reference and the inflection was designed as a sharp edge. Contours 2 and 3 were designed with a radius of $15 \mathrm{~mm}$, resp. $30 \mathrm{~mm}$ linking base nozzle and extension. Contour 4 is a combination of the larger radius with a sharp edge (half angle of the reference contour). Contour 5 is the opposite combination: a sharp edge followed with a radius. The various geometries of the inflection are represented figure 3 and summarized in table 1.
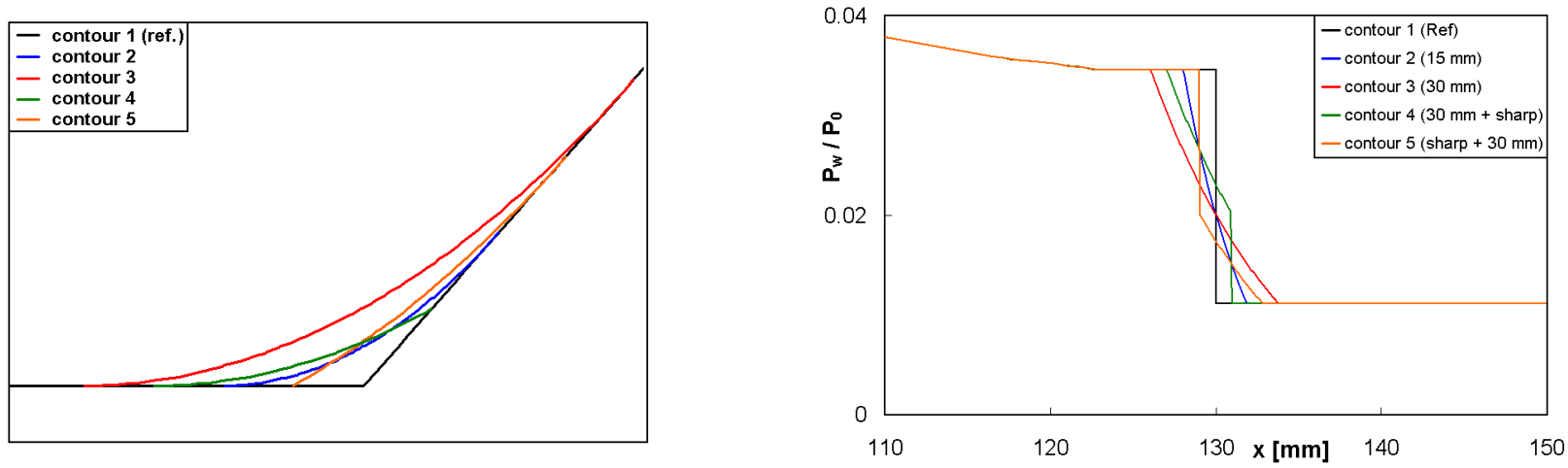

Figure 3. Geometries of the different contours and theoretical pressure distribution in the region of the inflection.

Table 1. Geometry of the five contours.

\begin{tabular}{cc}
\hline Contour 1 & sharp edge (reference) \\
Contour 2 & $15 \mathrm{~mm}$ radius \\
Contour 2 & $30 \mathrm{~mm}$ radius \\
Contour 4 & sharp edge $+30 \mathrm{~mm}$ radius \\
Contour 5 & $30 \mathrm{~mm}$ radius + sharp edge \\
\hline
\end{tabular}

The reference contour features a base nozzle designed as an ideal nozzle in full length. The design Mach number is $M_{D}=2.8$. The nozzle extension features an inflection angle of $15^{\circ}$ with the base nozzle and was 
chosen as an isobar, leading to a constant pressure extension. Numerical studies ${ }^{7}$ have shown the favorable influence of a positive wall pressure gradient in the extension to limit the inflection region length, but for the present study, a constant pressure extension has been chosen to limit the number of influence parameters. The flow characteristics of the test facility M11 were chosen, i.e. air - water damp mixture for a total temperature of $T_{0}=1000 \mathrm{~K}$. The geometrical parameters of the nozzle are summarized in table 2 .

Table 2. Geometrical parameters of the planar dual bell nozzle model.

\begin{tabular}{rcc}
\hline Throat radius & $R_{t h}$ & $8 \mathrm{~mm}$ \\
Base length & $L_{b} / R_{t h}$ & 16.25 \\
Extension length & $L_{e} / R_{t h}$ & 15 \\
Area ratios & $\epsilon_{b}$ & 3.95 \\
& $\epsilon_{e}$ & 7.97 \\
Inflection angle & $\alpha$ & $15^{\circ}$ \\
\hline
\end{tabular}

All of the models used for this study present a similar geometry, only the region of the inflection varies from one contour to the other.

\section{II.C. Instrumentation and data acquisition}

The flow behavior during the tests was recorded with wall pressure sensors and schlieren optics. The pressure ports were placed along the upper nozzle contour. Thin steel pipes were welded into the nozzle wall to connect the sensors with the $0.5 \mathrm{~mm}$ diameter orifices in the wall via small Teflon tubes. The repartition of the pressure ports in the region of the inflection was very dense to capture the evolution of the separation point for the different geometries.

A window of $45 \mathrm{~mm}$ diameter was perforated in the side plates of the model, in the region of the contour inflection. The flow evolution in this area could be observed using schlieren optics. The transient tests conducted at the cold flow facility P6.2 were recorded with color schlieren film. The variation of the feeding pressure, inducing the up- and down ramping of the NPR, led to the evolution of the flow in the nozzle models, from one operating mode to the other.

\section{Results and discussion}

The first campaign conducted for this study was the cold flow test series. The NPR was varied by up- and down-ramping of the feeding pressure. The nozzle model was designed specifically for warm flow conditions. The cold flow conditions at P6.2 facility did not led to an actual transition. The separation point displacement in the extension was progressive and indicates a slight negative wall pressure gradient when using cold nitrogen as feeding gas.
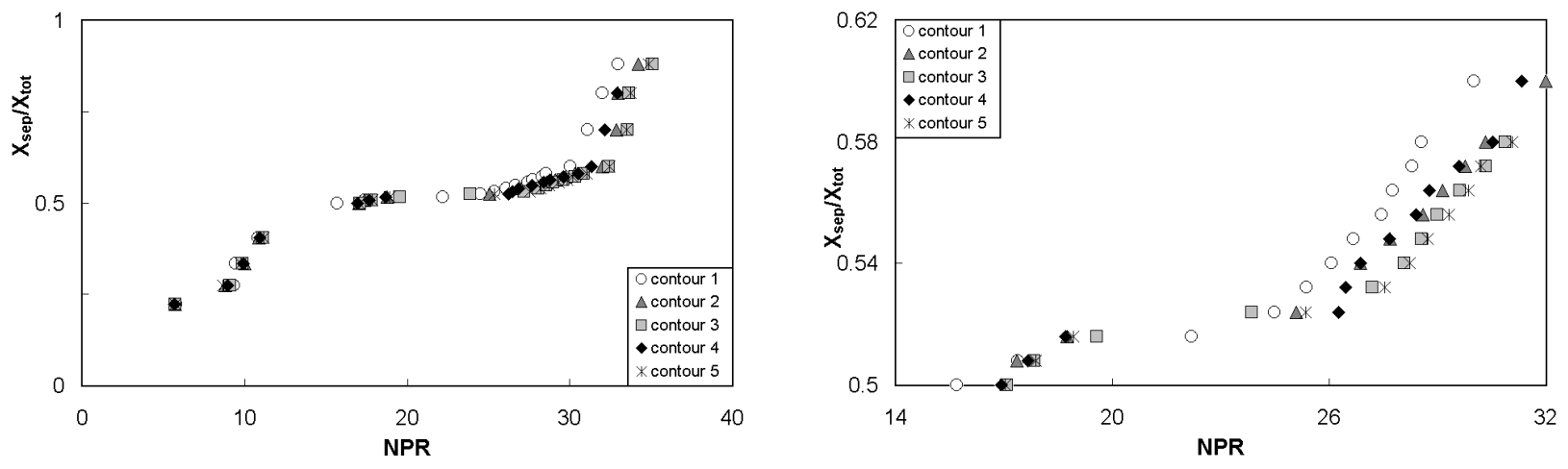

Figure 4. Position of the separation point as a function of the NPR value.

The axial position of the separation point in the nozzle is represented in Fig. 4: to each pressure port corresponds a NPR value. In a first step, the separation point moves in the base nozzle (up to NPR $=18$ ). Sea level mode is then reached and the separation point stays at the inflection while further increasing the 
NPR (NPR between 18 and 25). All five contours show a similar behavior since the geometrical differences only concern the region of the inflection.

The sneak transition starts when the flow separation reaches the inflection region, i.e. the first part of the extension, directly downstream the inflection, featuring a strong negative wall pressure gradient. This area is magnified in Fig. 4 (right) for a better resolution. Contour 1 leads to an earlier attachment of the flow in the inflection region $(\mathrm{NPR}=30)$. However, this effect is due to manufacturing issue, so that the contour with the sharp edge cannot be used as a reference. A former model, with similar contour, has to be used for comparison with the alternative geometries.

The flow reaches the end of the sneak transition the first for contour $4(\mathrm{NPR}=31)$ and the latest for contour 5 (NPR over 32). Once the transition has begun (in this case, it corresponds to the displacement of the separation point in the second part of the extension), the differences between the nozzle models decrease.

Figure 5 represents the wall pressure distribution in the vicinity of the inflection. The theoretical value of $P_{w}$ is given for contour 1. Typically, the length of the inflection region measured out of past experimental study is $L_{i} / R_{t h} \simeq 1$. Numerical simulations ${ }^{7}$ has show a higher value for the inflection length (between 1.8 and4), but for comparability reason, the experimental value is kept in the following.

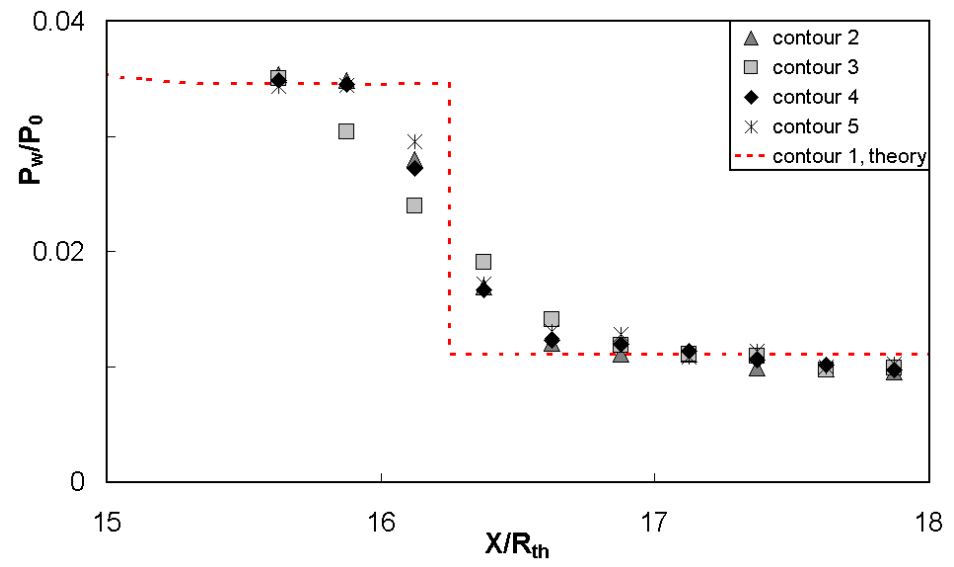

Figure 5. Wall pressure distribution in the vicinity of the contour inflection.

Contours 2 (small radius) and 4 (edge + radius) feature a value of $L_{i} / R_{t h}$ comparable to the reference. The inflection region length reaches 1.25 for contour 5 (radius + edge) and 1.5 for contour 3 (30 mm radius). The characteristics of the five contours are summarized in table 3. For contour 1 , the inflection region length is given from a former study, but unfortunately, no values are available for the NPR interval leading to sneak transition.

Table 3. Comparison of the flow behavior for the different inflection geometries.

\begin{tabular}{rcc}
\hline Contour name & $\begin{array}{c}\text { Inflection length } \\
L_{i} / R_{t h}\end{array}$ & $\begin{array}{c}\text { Sneak transition } \\
(\Delta \mathrm{NPR})\end{array}$ \\
Contour 1 & 1 & - \\
Contour 2 & 1 & 5.2 \\
Contour 3 & 1.5 & 7 \\
Contour 4 & 1 & 4.2 \\
Contour 5 & 1.25 & 5.8 \\
\hline
\end{tabular}

For the warm flow test campaign, three contours have been chosen. Unfortunately, the manufacturing problems of contour 1 were not yet known at that point, so that contour 1,3 and 4 were tested at M11 facility. Under the warm conditions for which the contours were designed, the actual transition took place.

The test facility M11 offers only steady state conditions. To investigate the transition condition, a series of operation points in total pressure and temperature have been chosen. For each point, the flow condition has been determined thanks to the wall pressure measurements. 


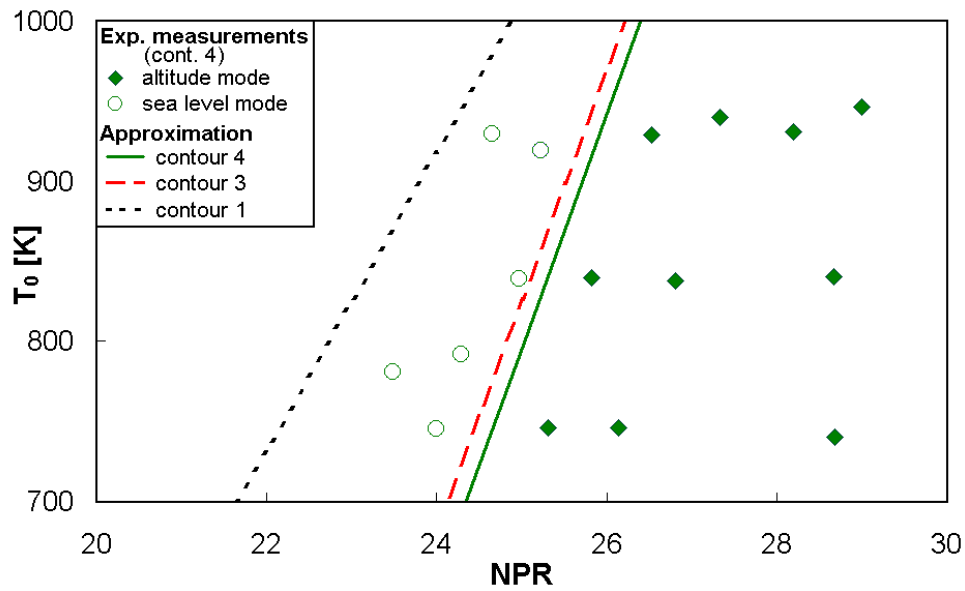

Figure 6. Separation conditions for the three contours tested under warm flow conditions

Figure 6 represents the flow mode obtained for the different operating conditions with contour 4 . The transition NPR can be approximated between the conditions of sea level and altitude modes. The linear approximation is given for contour 4. The relation obtained for contour 3 and 1 are also depicted for comparison. $N P R_{t r}$ increases with the total temperature of the flow. Contours 3 and 4 show a comparable behavior; the contour with the radius leads to slightly earlier transition. The evolution of the transition for contour 1 shows a significant deviation compared to the two other contours (lower $N P R_{t r}$ and more pronounced temperature influence), but this effect is, once again, due to the manufacturing uncertainties.

The wall pressure distribution in nozzle contours 3 and 4 is given in Fig. 7. The measurement in cold and warm flow conditions are compared with the design pressure distribution.
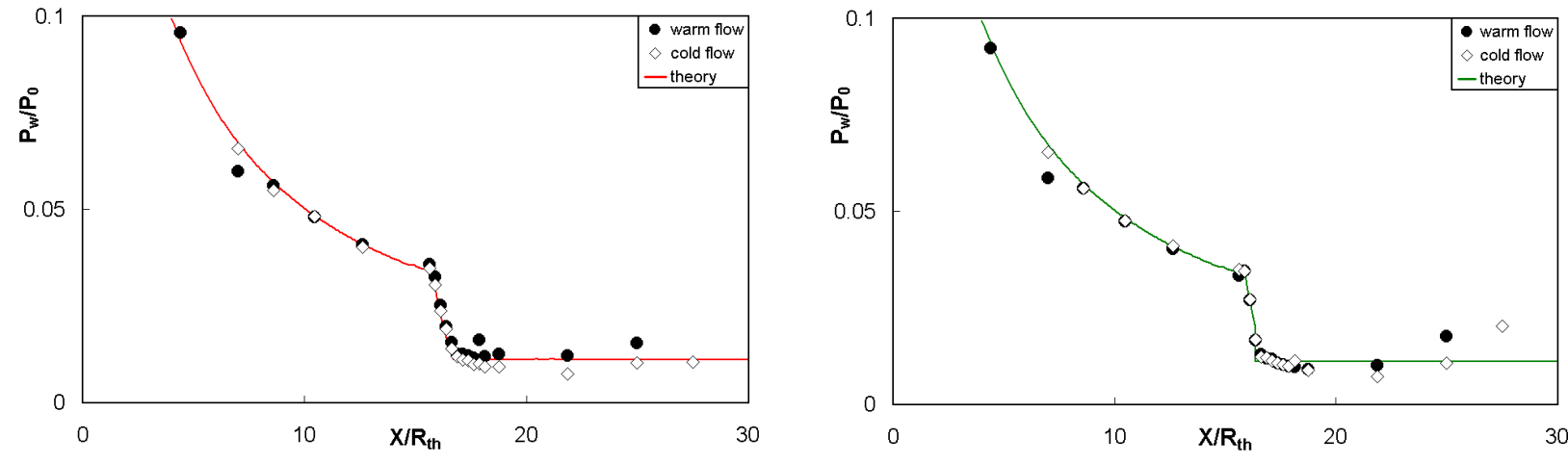

Figure 7. Wall pressure distribution in warm and cold flow conditions for contour 3 (left) and 4 (right).

The measurements under warm flow conditions are slightly higher than the cold flow values, in particular in the extension. The large wall thickness $(10 \mathrm{~mm})$ of the models are not sensible to thermal deformations. The difference in pressure measurements is mainly due to the difference of flow gas mixture. The lower wall pressure in the extension may explain the absence of an actual transition in cold flow tests.

Contour 3 was the first contour to be tested on M11 facility. The total temperature was chosen between $600 \mathrm{~K}$ and $1000 \mathrm{~K}$. Figure 8 represents the flow mode reached for each operating point. For the lowest temperature, the NPR transition from one mode to the other is significantly lower than for the other temperatures.

At $600 \mathrm{~K}$, the water molecules present in the flow starts to condense. The temperature of the flow increases due to the release of vaporization heat. Figure 9 represent the wall pressure distribution in the base nozzle of contour 3 for a total temperature of $600 \mathrm{~K}$ and $700 \mathrm{~K}$. In the presence of condensation, the wall pressure increases significantly.

It has been shown by Oswatitsch ${ }^{8,9}$ that condensation in supersonic nozzle starts after the flow temperature has fallen $20 \mathrm{~K}$ to $50 \mathrm{~K}$ under the evaporation line. The condensation of the water in the flow leads to 


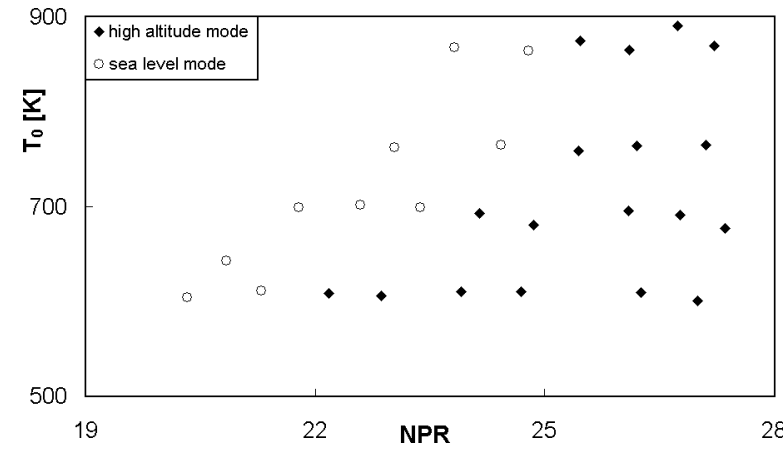

Figure 8. Transition condition of the nozzle model 3 at M11.

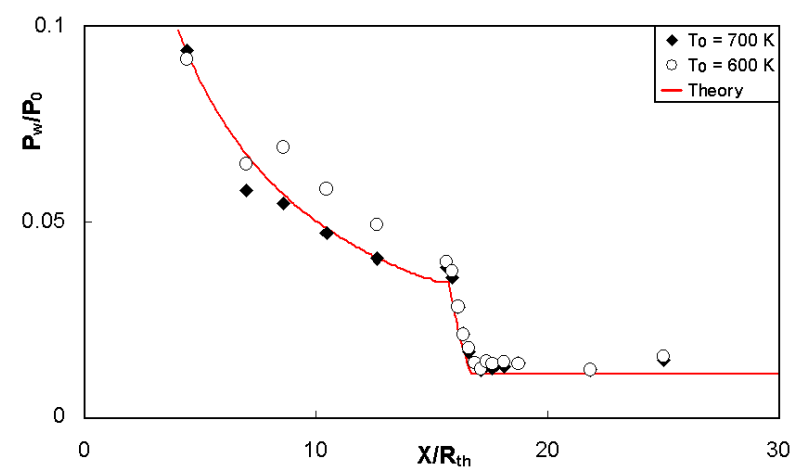

Figure 9. Wall pressure distribution in contour with and without condensation effect.

a pressure increase at the base nozzle wall. So that the transition conditions are reached earlier at the base nozzle end, leading to a smaller value of $N P R_{t r}$. For the two other contours, the tests were conducted only for total temperature between $700 \mathrm{~K}$ and $1000 \mathrm{~K}$.

\section{Conclusion}

An experimental study has been conducted under cold and warm flow conditions on five dual bell nozzles featuring different inflection geometries. Although one of the model presented manufacturing issues, the comparison of the results has shown an increased inflection region for a large radius and the combination of radius and edge. The contour featuring a combination of sharp edge and radius seems to be the most promising alternative with the shorter NPR interval leading to sneak transition for the same inflection region length as the original geometry. The transition NPR increases with the flow temperature in a similar way for contour 3 and 4 . The exploitation of the schlieren pictures should bring further information on the evolution of the transition from one mode to the other.

\section{Acknowledgments}

Financial support has been provided by the German Research Foundation (Deutsche Forschungsgemeinschaft - DFG) in the framework of the Sonderforschungsbereich Transregio 40.

\section{References}

\footnotetext{
${ }^{1}$ Foster, C. and Cowles, F., Experimental study of gas-flow separation in overexpanded exhaust nozzles for rocket motors, Progress Report, 4-103, Jet Propulsion Laboratory, 1949.

${ }^{2}$ Horn, M. and Fisher, S., Dual-Bell Altitude Compensating Nozzles, NASA-CR-194719, 1994.

${ }^{3}$ Hagemann, G., Immich, H., Van Nguyen, T. and Dumnov, G., Advanced Rocket Nozzles, Journal of Propulsion and Power, 1998.

${ }^{4}$ Nürnberger-Génin, C. and Stark, R., Flow transition in dual bell nozzles, Shock Wave Journal, Vol. 19, No. 3, pp. 265-270, 2009.

${ }^{5}$ Génin, C. and Stark, R. Side loads in sub-scale dual bell nozzles, Journal of Propulsion and Power, Vol. 27, No. 4, July/Aug. 2011.

${ }^{6}$ Genin, C. and Stark, R. Hot flow testing of dual bell nozzles, 49th Aerospace Science Meeting, 4-7 January 2011, Orlando, FL, 2011.

${ }^{7}$ Martelli, E., Nasuti, F. and Onofri, M. Numerical parametric analysis of dual-bell nozzle flows, AIAA Journal, Vol. 45, No.3, March 2007.

${ }^{8}$ Frank, W. Condensation phenomena in supersonic nozzles, Acta Mechanica, Vol. 54, pp 135-156, 1985.

${ }^{9}$ Frey, M. Test case 1c: Side loads in DLR TIC nozzle, ATAC-FSCD Workshop "Afterbody and nozzle flows", November 14th, 2006.
} 